\title{
Out of band radiation effects on resist patterning
}

\author{
Simi A. George* and Patrick P. Naulleau \\ Center for X-Ray Optics, Lawrence Berkeley National Laboratory, Berkeley, CA 94720 \\ *Now at SCHOTT North America, Duryea, PA 18642
}

Email: simi.george@us.schott.com and pnaulleau@lbl.gov

\begin{abstract}
Our previous work estimated the expected out-of-band (OOB) flare contribution at the wafer level assuming that there is a given amount of $\mathrm{OOB}$ at the collector focus. We found that the OOB effects are wavelength, resist, and pattern dependent. In this paper, results from rigorous patterning evaluation of multiple OOB-exposed resists using the SEMATECH Berkeley 0.3-NA MET are presented. A controlled amount of OOB is applied to the resist films before patterning is completed with the MET. LER and process performance above the resolution limit and at the resolution limits are evaluated and presented. The results typically show a negative impact on LER and process performance after the OOB exposures except in the case of single resist formulation, where resolution and performance improvement was observed.
\end{abstract}

Keywords: EUVL, out-of-band, resists, flare, blur, aerial image modeling

\section{INTRODUCTION}

Plasmas have temperature gradients that produce numerous excited states which radiate into a large range of wavelengths. Extreme ultraviolet lithography (EUVL) systems will use incoherent, tin based plasma light sources for the $13.5 \mathrm{~nm}$ illumination with a $2 \%$ bandwidth. The high reflectivity, Mo-Si multilayer coated optics that are necessary for band selection in EUVL systems have also been shown to be efficient reflectors for longer wavelengths [1-3]. The calculated reflectivity for a Mo-Si multilayer (40 layer pairs) and the reflectivity of the Ruthenium capping layer is given in Fig. 1 (near normal incidence, $5^{\circ}$. It is shown that these multilayer coatings have high reflectivity for wavelengths greater than $150 \mathrm{~nm}$.

In addition to the high reflectivity of the multilayer optics to long wavelengths, the resists that have been developed for EUVL utilize constituents that can be sensitized by ultraviolet/deep ultraviolet (UV/DUV) exposures. Some of the EUV resists that are currently being utilized for patterning studies have been shown to be many times more sensitive to specific wavelengths in the 150-300nm DUV/UV region as compared to the sensitivity at $13.5 \mathrm{~nm}$ [4]. This is of significant concern since the tin plasma based EUV light sources are known to produce quantifiable emission in this region along with the required source bandwidth. Moreover, a part of these additional emissions from the source can be collected and transmitted to the wafer level resulting in the unwanted background exposure of the resists (flare) resulting in reduced image contrast when patterning. In this paper, we use out-of-band (OOB) to refer to only those wavelengths that the resists are most sensitive to and this is the region between $150 \mathrm{~nm}-300 \mathrm{~nm}$. The effective flare contribution from $\mathrm{OOB}$, in addition to the flare resulting from the low spatial frequency roughness scatter of the optics, is expected to be significant.

Our previous work outlined a simple method for estimating the expected OOB flare contribution at the wafer level by assuming that there is a given amount of OOB at the collector focus. By taking into account resist sensitivities for a single resist at certain key wavelengths, we found the flare contribution to be approximately 4-5\%. Further details on these calculations can be found in reference[5]. With further modeling, we found that the OOB effects are wavelength, resist, and pattern dependent. A brief overview of these results is presented in the following section. In this paper, we 
focus on the data collected from the rigorous resist patterning studies on multiple EUV resists using the SEMATECH Berkeley 0.3-NA MET [6] and controlled amounts of OOB radiation. Detailed process evaluation on the impact of selected OOB radiation, above and below the resolution limit, on two champion EUV resists is presented.

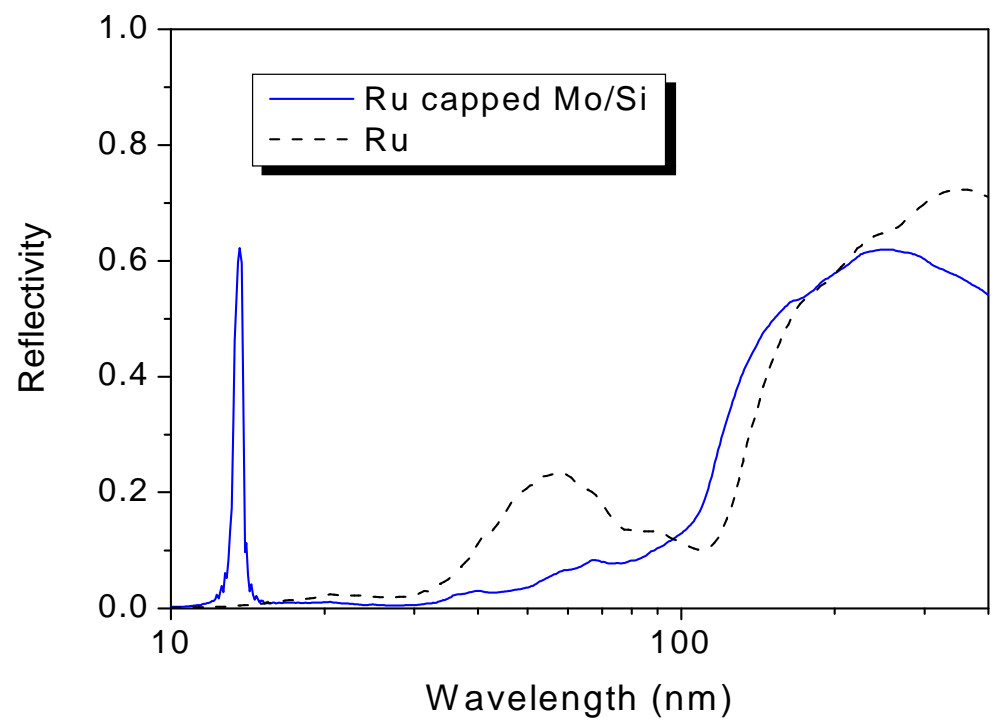

Figure 1. (Left) figure shows the near normal incidence reflectivity $\left(5^{\circ}\right)$ for $\mathrm{Ru}$ and for a standard $\mathrm{Ru}$ capped $\mathrm{Mo} / \mathrm{Si}$ multilayer. The reflectivity was calculated using optical constants from the Handbook of Optical Constants of Solids[2,3] (Data courtesy of Dr. Eric Gullikson).

\section{OOB RELATED FLARE ESTIMATIONS AT THE IMAGE PLANE}

A simple method for estimating DUV/UV contribution from sources in addition to the EUV dose at the image plane was outlined previously[5]. Resist dependant flare was calculated with supplier provided OOB wavelength specific resist sensitivity values as compared to the published absolute EUV sensitivity[7] for a resist. This particular resist was more sensitive to $157-\mathrm{nm}$ radiation than other wavelengths. The effective fare was calculated by dividing the $100-400 \mathrm{~nm}$ region into four bands. A summary of the results is provided in Table 1. The starting point for all calculations is the collector focal point of the lithography exposure optical system called the intermediate focus[8].

\subsection{ADT flare test features modeled as a benchmark}

In the previous calculations, flare from OOB is treated as a uniform background (DC) problem. This was believed to be less accurate, since the shorter wavelengths in the DUV can image the large features on the mask. Furthermore, it can be expected that the effective flare and the resulting contrast loss for patterning may vary with feature types, sizes, density, and with the tone of the mask. In order to evaluate such effects, a simple in-house developed, two dimensional point spread function (PSF) model is utilized.

To benchmark the modeling methodology, mask features that were used in a set of measurements completed at the ASML alpha demo tool (ADT) to estimate OOB is used [9]. For these measurements, varying number of reflections from reticle masking blades were used while imaging a circular absorber post of 200-nm diameter surrounded by a bright area on the mask. The diameter of the bright area was varied, while the absorber diameter was kept constant. It was found that the contributions from flare increased for larger bright area diameters. The measurements led to the estimate that there is approximately $4 \% \mathrm{OOB}$ radiation at the image plane. The two dimensional modeling methodology was also verified against rigorous three dimensional vector modeling using Panoramic's EM-Suite software[10] demonstrating good agreement between the two methods. 
The results from the calculations using the same mask features and exposure system parameters are given in Fig. 2 . Details of the modeling methods can be found in reference [11]. A saturation effect is found as the bright areas become much larger than the $200-\mathrm{nm}$ post feature as was observed in the measurements.

Table 1: Effective flare calculated from OOB wavelength specific resist sensitivity as related to the EUV sensitivity

\begin{tabular}{|c|c|c|c|}
\hline $\begin{array}{c}\text { Wavelength } \\
(\mathbf{n m})\end{array}$ & Sensitivity Factor & $\begin{array}{c}\text { 11 Mirrors, 10\% } \\
\text { OOB } \\
\text { at IF (\%) }\end{array}$ & $\begin{array}{c}\text { 10 Mirrors, 10 \% at } \\
\text { IF (\%) }\end{array}$ \\
\hline$* 13.5$ & $\mathrm{X}$ & 100 & 100 \\
\hline 157 & $4 \mathrm{x}$ & 3.15 & 3.74 \\
\hline 193 & $0.24 \mathrm{x}$ & 0.35 & 0.42 \\
\hline 248 & $\mathrm{X}$ & 0.83 & 1.01 \\
\hline \multicolumn{2}{|c|}{$\begin{array}{c}\text { Total Effective OOB flare on wafer } \\
\text { (Ru capped MLM) }\end{array}$} & $\mathbf{4 . 3 4}$ & $\mathbf{5 . 1 7}$ \\
\hline
\end{tabular}

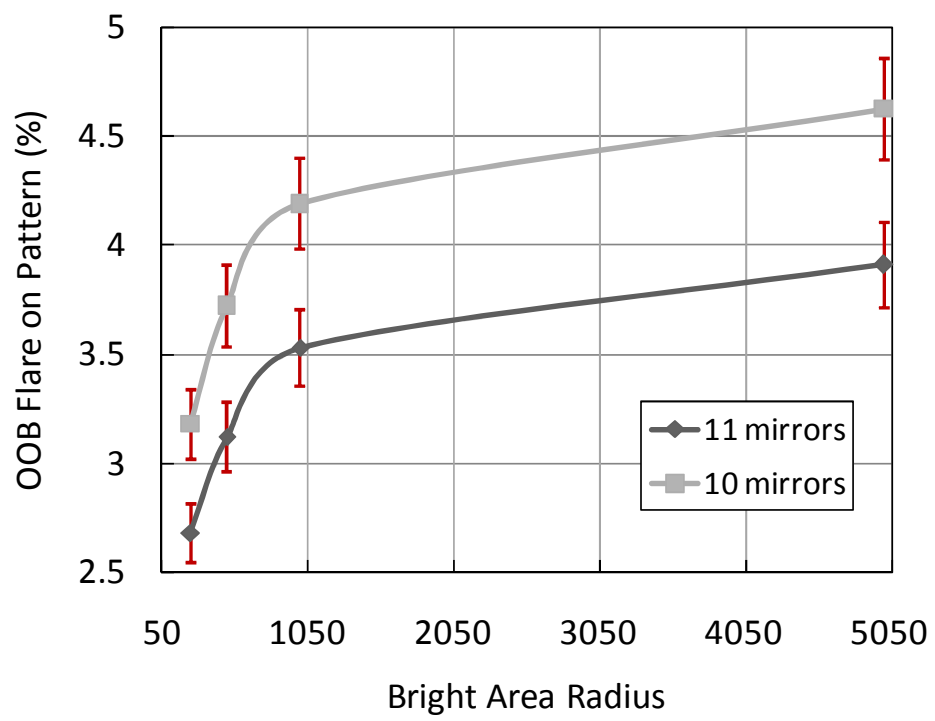

Figure 2. Plot of the tabulated OOB flare (table 3). Saturation in the flare may be expected as the bright areas become infinitely large in comparison to the $200 \mathrm{~nm}$ absorber post. A very large bright area was not calculated for computational reasons.

The model used here is believed to give a first order approximation of the effective flare on features resulting from the resist sensitive wavelengths reaching the wafer plane. It is worth noting that these values are estimated based on one set of resist parameters and neglecting any other effects such as mask scattering, changes in absorber reflectivity, diffraction effects, pitch dependencies, etc. Since all of the aerial image simulations are completed in image space and since these features are much larger in comparison to the critical dimensions required, it is expected that we maybe underestimating the flare effects. The next section outlines results for several critical dimension mask features using the same methodology.

\subsection{Feature specific flare estimates with the 2D model}

In the same way that was described before, feature specific evaluations are completed for flare effects at the 22-nm half pitch. Absorber effects are examined for brightfield and darkfield masks with isolated and dense, line and contact 
features. Typical production type system parameters with $0.32-\mathrm{NA}, 0.5 \sigma$ conventional illumination are used. Figure 3 gives examples of the mask types for the 22-nm line/space CD. Significant differences in contrast are observed between the bright and dark masks for the same line width. Some mitigation of flare with darkfield masks is seen with an isolated feature. Dense features show proximity effects and drastic variations in contrast depending on the feature types. The impact on 2D features like contacts are much stronger than the lines and spaces only patterns as can be seen from Fig. 4. 3D calculations using commercially available EM-Suite that account for diffraction effects and 3D effects did not show significant differences from the method that is used here.
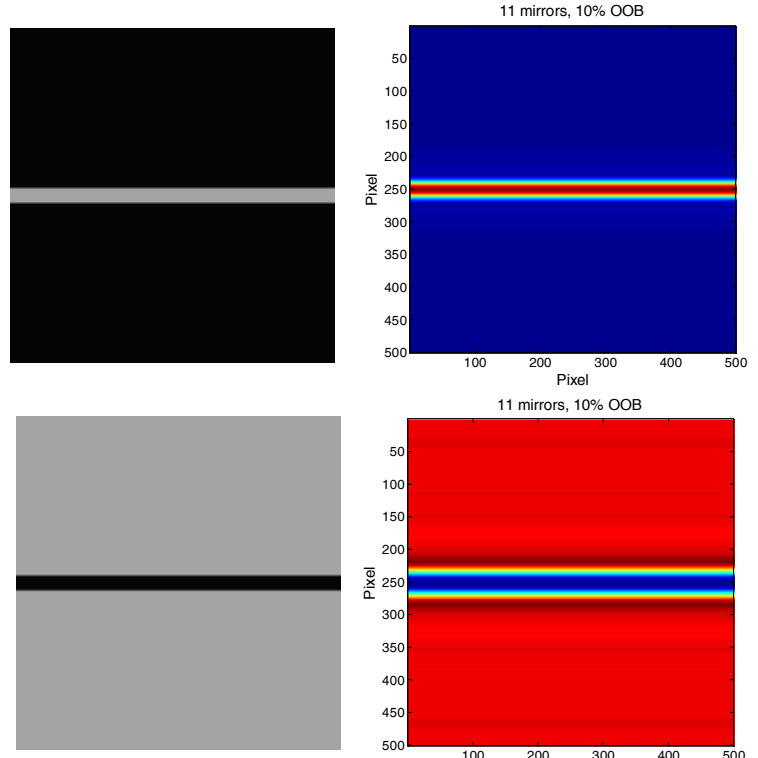

Mask

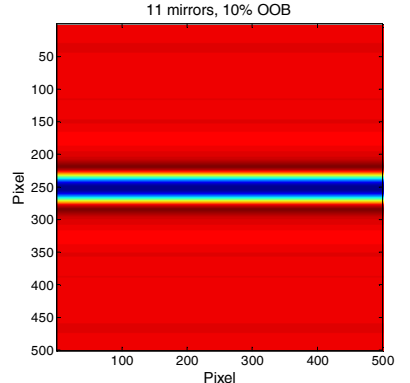

Aerial Image

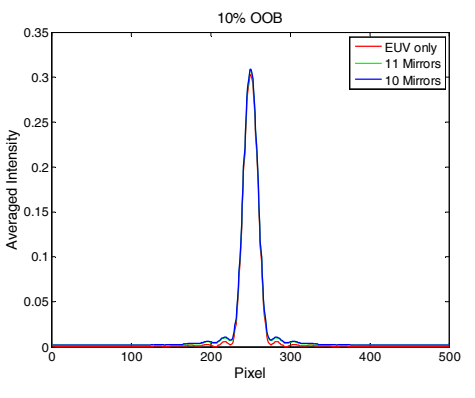

$1.72 \%$

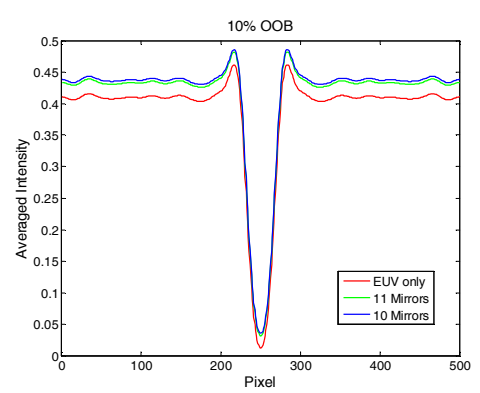

$5.42 \%$

Figure 3. OOB effects over EUV imaging for a 22nm, isolated bright and dark line. Intensity profiles show differences in the two cases. The flare calculated for an 11 mirror system is shown on the left, much more on the data is in reference [11].
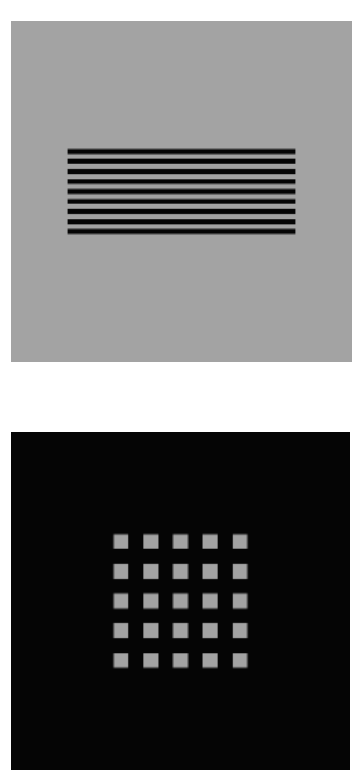

Mask
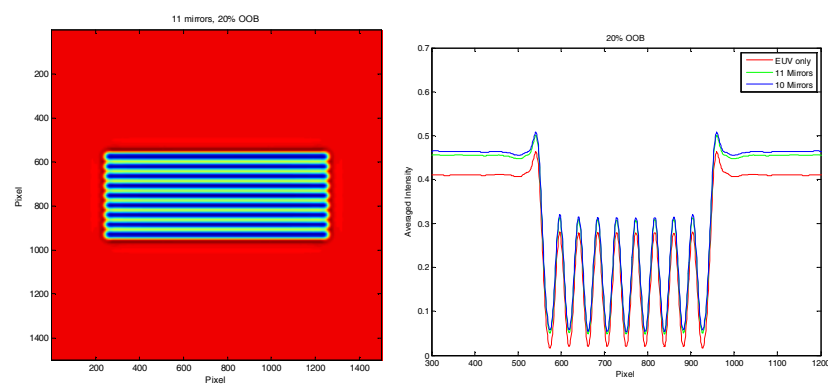

$\mathbf{5 . 0 4 \%}$

$4.00 \%$

in Dark-field
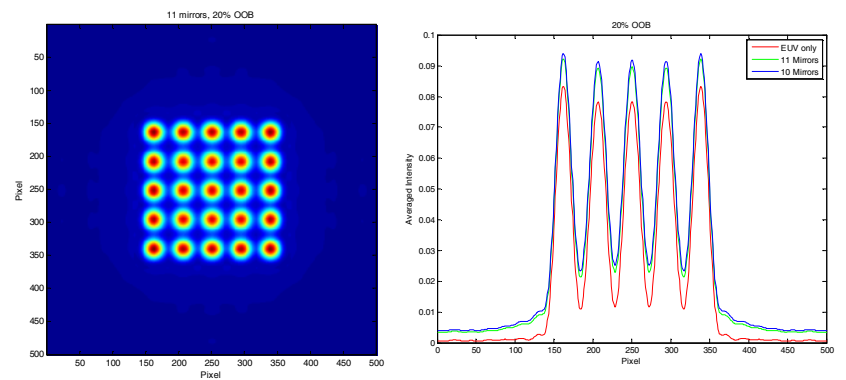

$7.23 \%$

$11.23 \%$

in Bright-field

Aerial Image

Figure 4. OOB effects over EUV imaging for 22nm, dense lines and contacts. Intensity profiles show significant differences in the two cases as compared. The flare on 2D features are much greater even in a dark field as shown on left for 11 mirrors. 


\section{OOB EXPOSURES AT THE MICRO-EXPOSURE TOOL}

All of the resist patterning is completed with the SEMATECH Berkeley 0.3 NA, EUV micro-field exposure tool (MET) installed at the Advanced Light Source synchrotron facility at the Lawrence Berkeley National Laboratory. The MET uses spectrally pure, debris-free, undulator radiation as the source of EUV radiation. The programmable coherence illumination capabilities provide imaging down to $12 \mathrm{~nm}$. Details on the MET can be found in the literature[6]. The OOB imaging studies were completed by pre-exposing resists coated on a 4-inch wafer, with an external DUV/UV source of sufficient brightness and uniformity.

\subsection{Out-of-Band Exposure Methods}

The source used for these studies is the Energetiq LDLS source [12]. Figure 5 shows the mechanical design completed for incorporating the source into the MET. Optical design was completed using ZEMAX and toroidal mirrors are utilized for light collection and beam shaping. The DUV light source is currently set-up as shown in Fig. 5 on an optical bench and radiation is focused onto resist films coated on 4-inch wafers. The calibrated, spectral distribution from this source for available wavelengths of interest to this study is shown in Fig. 6 (left) for a $1 \mathrm{~mm}$ aperture at the source output. The measured power output at the two key resist sensitive wavelengths of $193 \mathrm{~nm}$ and $248 \mathrm{~nm}$ are $0.04 \mathrm{~mW} / \mathrm{nm}$ and 0.09 $\mathrm{mW} / \mathrm{nm}$, respectively. Wavelength selection for the exposures is implemented with filters obtained from Acton Optics \& Coatings[13]. These filters have approximately $24 \mathrm{~nm}$ of FWHM bandpass (Fig. 6, right). Filters are aligned into the optical path and interchanged using a fly wheel. With this configuration, controlled resist exposures can be completed with three illuminations; the $193 \mathrm{~nm}$ band, the $248 \mathrm{~nm}$ band, and in the broadband.

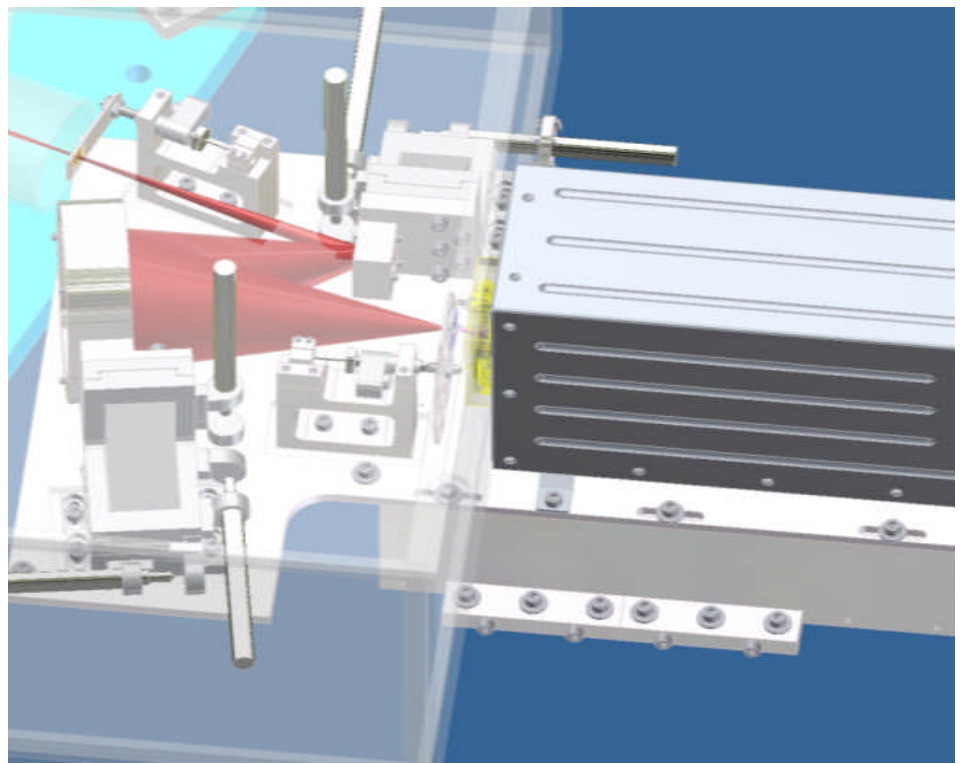

Figure 5, (Left) Current table top set-up for the pre-exposure of resists with bandwidth selected OOB wavelengths. (Right) Source spectral output provided by the source supplied. Wavelengths from 170-290nm are available.

For exposures, the DUV beam is collected, focused, and apertured to a uniform $8 \mathrm{~mm} \times 10 \mathrm{~mm}$ area. An SXUV IRD photodiode [14] with an active area of $10 \mathrm{~mm} \times 10 \mathrm{~mm}$ is used for dose measurements. Close to linear responsivity of this photodiode to the DUV wavelengths makes it ideal for these measurements. Dose measurements are taken prior to each exposure to monitor any possible changes. The resist coated wafer is aligned to the DUV beam inside a high purity Nitrogen purged chamber and exposed. The exposed wafer is removed and placed into a desiccator and vacuum sealed for transport to the MET. Care is taken to minimize any contact of the samples to non-clean room environment after DUV exposures. EUV patterning is completed over the DUV exposed area on the wafer with keeping all other process conditions as similar as possible. 

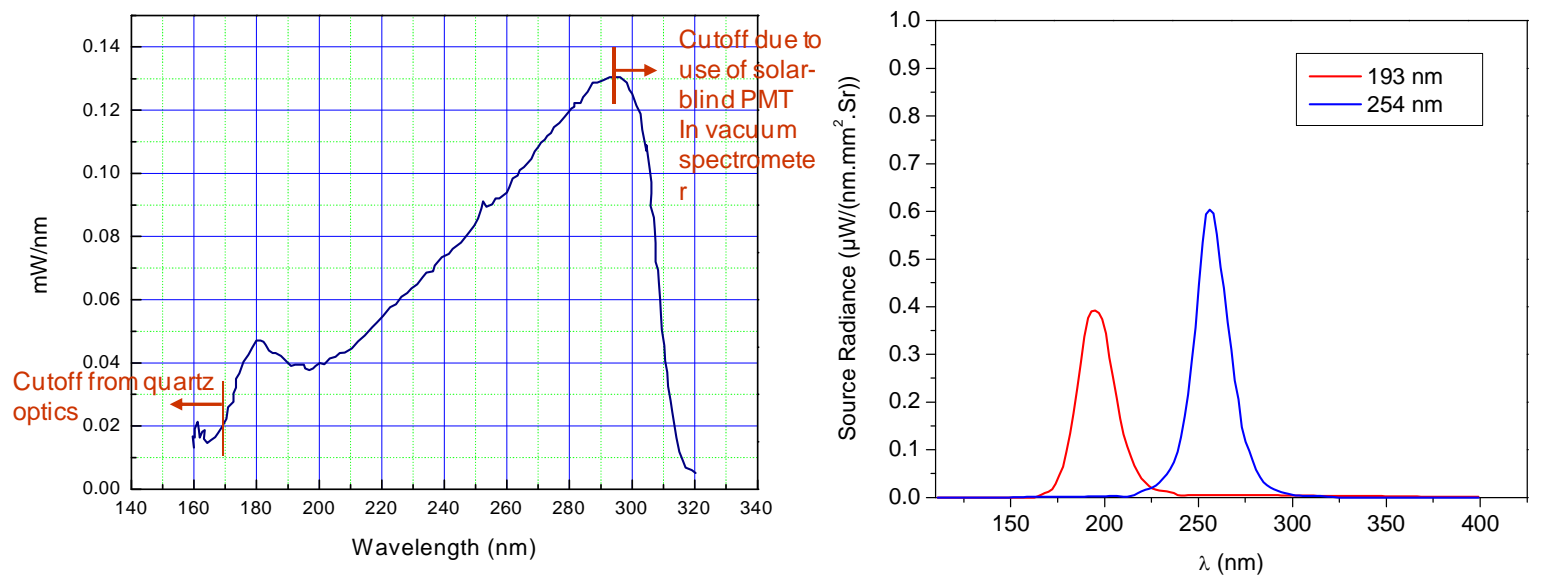

Figure 6, Calibrated, spectral distribution from the Energetiq LDLS light source (left) for a $1 \mathrm{~mm}$ aperture at source output. 193-nm and 248-nm wavelength selection for the OOB exposures is completed with filters obtained from Acton Optics \& Coatings with approximately $24 \mathrm{~nm}$ of FWHM bandpass ( right).

\subsection{Resist Exposures}

A number of high resolution, chemically amplified EUV resists, that are typically used as baseline resists for the MET, were tested for these studies. Two of these resists, BBR08A and BBR08B, were tested multiple times for repeatability.

For most tests, the OOB exposure is limited to nominally $10 \%$ of EUV dose required for clearing the pattern of interest for a given resist. For these studies we chose the EUV dose to clear 50-nm half-pitch lines and spaces, since this is well above the resolution limit of these resists. All experiments shown here are completed with the MET baseline illumination; annular with 0.35/0.55 sigma. Standard resist process conditions prescribed for EUV exposures are used for all of the samples.

The film thickness used for both resists is $50 \mathrm{~nm}$. BBR08A was coated on Hexamethyldisilazane (HMDS) primed Silicon wafer for results given in this paper. BBR08B was evaluated on HMDS as well as on a bottom anti-reflective coating (BARC) that is typically used for studies at the MET. A 40-nm BARC coating was applied. Measurement results comparing the $193 \mathrm{~nm}$ band, $248 \mathrm{~nm}$ band, and the broadband illumination related performance effects for both resist are given in the following section.

\section{RESULTS: OOB EXPOSURE EFFECTS ON EUV RESISTS}

The exposed and developed resist patterns are imaged using the Hitachi ultra-high resolution field emission scanning electron microscope model S-4800[15]. SEM images of 50-nm half-pitch vertical lines was collected for a number of exposure steps through focus. Smaller half-pitch data was also collected as needed. Detailed image analysis of process performance and line edge roughness (LER) measurements are completed with the commercially available software, SuMMIT[16].

\subsection{Error baseline for the MET patterning studies}

To make sure that what is observed is real and beyond shot-to-shot process noise, we compiled data for multiple wafer printing over an 8-hour period for BBR08A. Using the image data through focus and dose for five patterned wafers, the measurement uncertainty on exposure latitude and LER is determined. The exposure latitudes generated from the dosefocus matrix is given in Fig. 7. The dose variation is approximately 6\%, 3-sigma LER variation is $0.3 \mathrm{~nm}$, and focus changes can be between $50 \mathrm{~nm}$ and $100 \mathrm{~nm}$ for wafer to wafer patterning and process. If the changes in patterning that are observed with OOB exposures are greater, then it can be considered as real. 


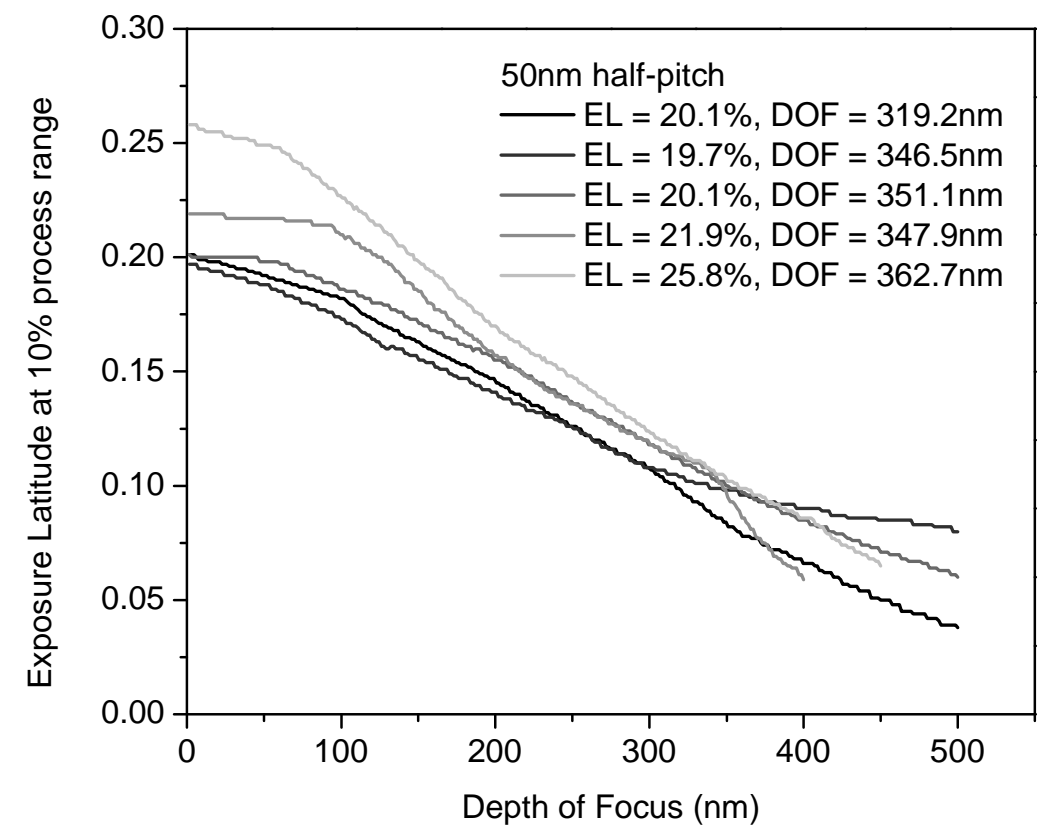

Figure 7. Wafer to wafer process baseline for BBR08A patterned with the SEMATECH Berkeley 0.3NA MET. From this data set, we find that the dose variation is approximately $6 \%, 3$-sigma LER variation is $0.3 \mathrm{~nm}$, and focus changes can be between $50 \mathrm{~nm}$ and $100 \mathrm{~nm}$, though the absolute change depth of focus (DOF) is found to be only $40 \mathrm{~nm}$.

\subsection{OOB effects on BBR08A}

The resist coated films are taken out of the amine filtered environment in order to complete the OOB exposures before patterning with MET, for these studies. For that reason, the patterning on a resist film that was exposed only to the OOB environment for the duration of a typical a wafer exposure experiments was compared to the standard EUV only patterning results. Figure 8 compares focus-exposure data collected for resist patterns on these two wafers. No major changes are observed; iso-focal dose is the same in both cases, EL computed is well within the 6\% error bar, a focus shift of $50 \mathrm{~nm}$ is observed (within the expected uncertainty), and the averaged LER computed for the best area of the process window shows a difference of $0.22 \mathrm{~nm}$ between the two wafers (again smaller than the uncertainty), leading to the conclusion that for this resist, the environment is not a concern.
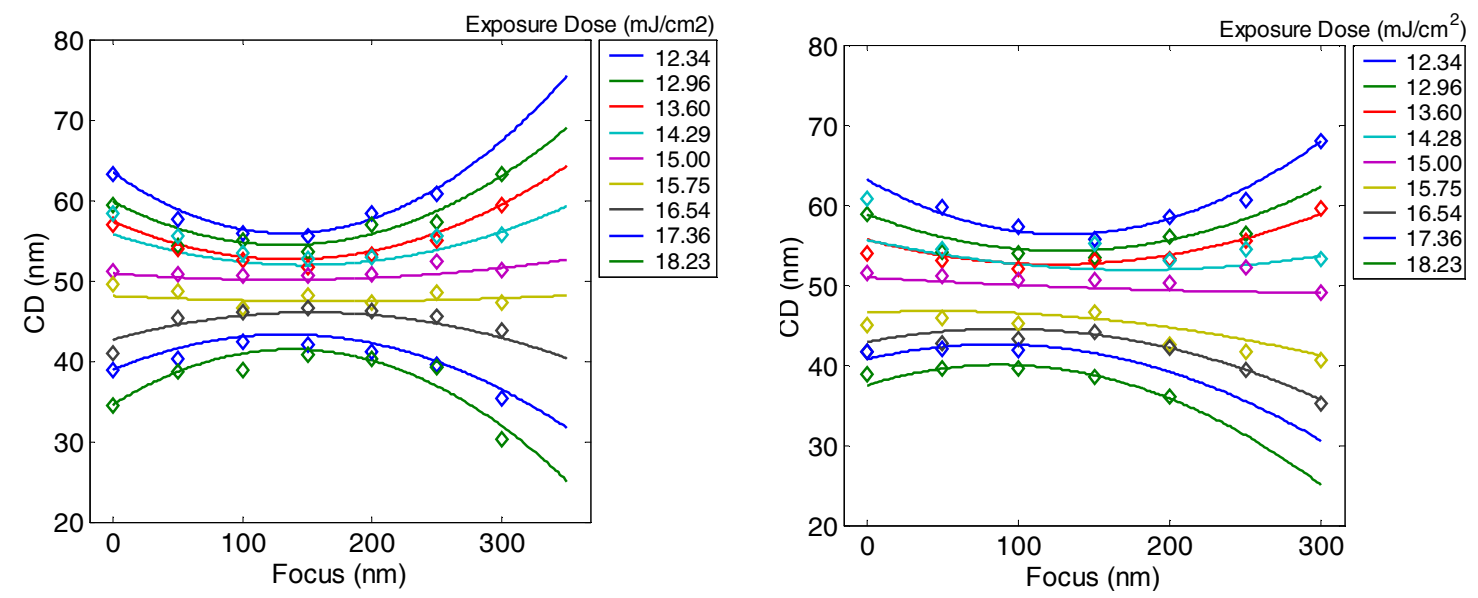
Figure 8: Process window comparison for isolating for any effects on resist films due to environmental contamination possible during the OOB exposures.

Process window and LER data for EUV only patterning is compared to the broadband exposure on BBR08A in Fig. 9. Resist photospeed appears to be impacted. The collected data appears to be overdosed. The LER corresponding to the best process area is increased by more than a nanometer. We find the $193 \mathrm{~nm}$ and $248 \mathrm{~nm}$ illumination to give similar results in the case of this resist. The impact on LER is much more significant near the resolution limit as can be seen from Fig. 10.
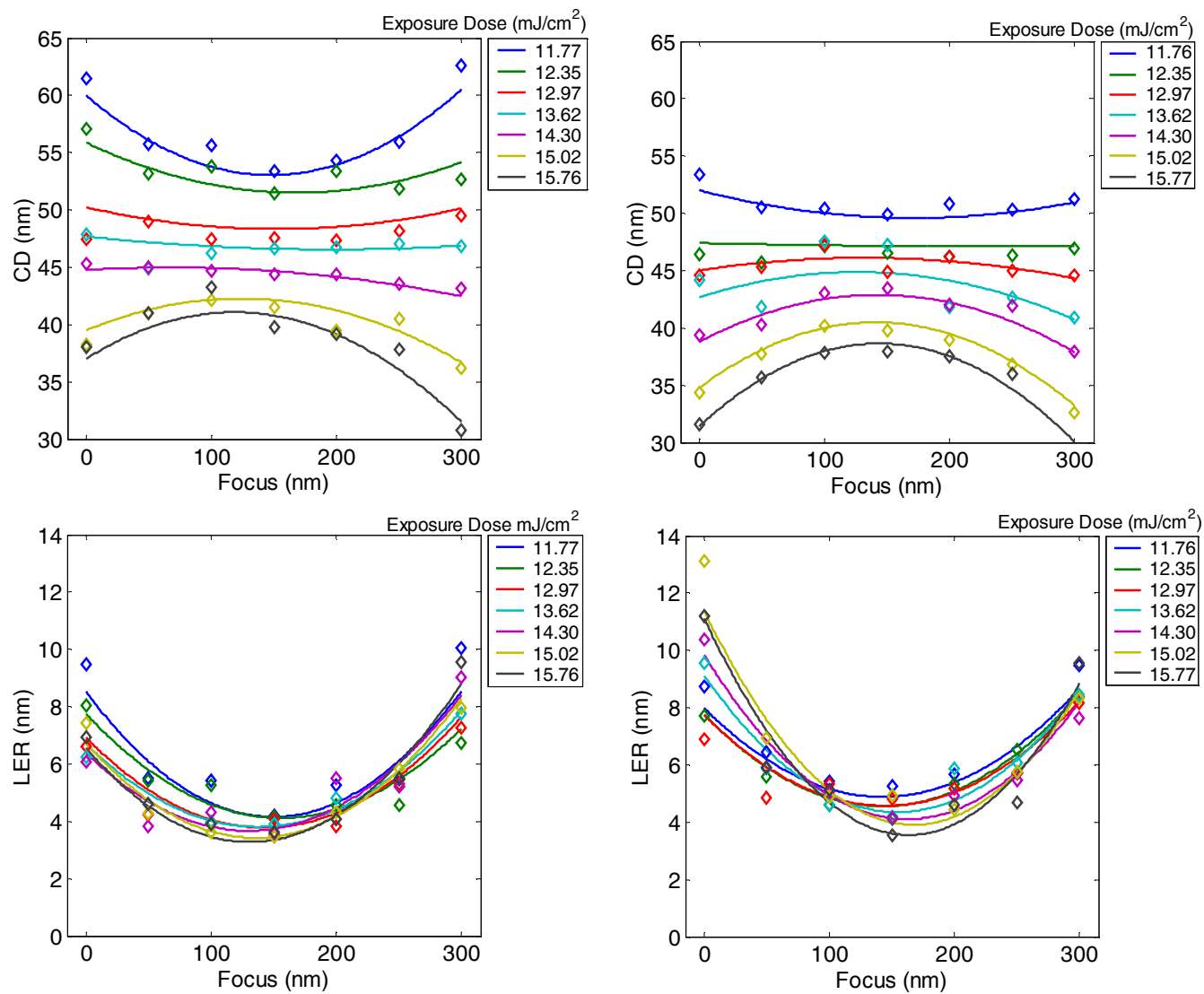

Reference, EUV only, Isofocal Dose $=13.62 \mathrm{~mJ}$ Isofocal $\mathrm{CD}=47 \mathrm{~nm}$, LER average $=4.1 \mathrm{~nm}$

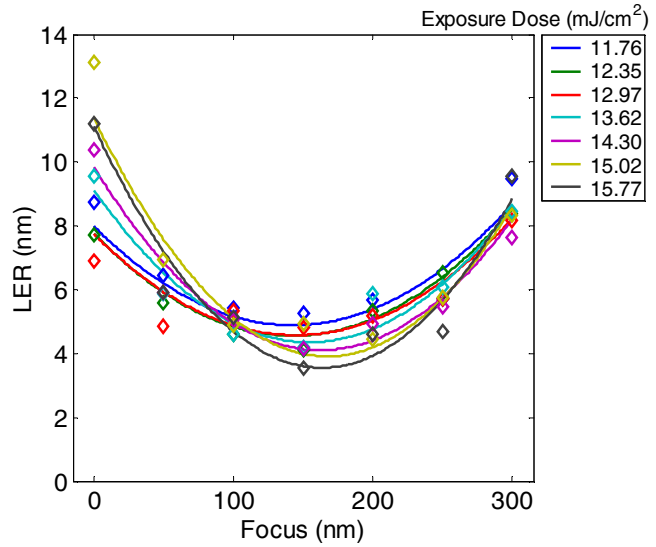

EUV only + broadband $\left(1.25 \mathrm{~mJ} / \mathrm{cm}^{2}\right)$ Smaller Dose shift, LER is $5.2 \mathrm{~nm}$

Figure 9. Process map and LER measurements corresponding to the process data is shown for EUV only patterning (left) and broadband exposed patterns (right) for BBR08A. LER performance is significantly worse and changes in photospeed is seen.

\begin{tabular}{|c|c|c|c|}
\hline $\begin{array}{c}\text { Reference: EUV only } \\
\text { CD }=29.0 \mathrm{~nm} \\
\mathrm{LER}=4.5 \mathrm{~nm}\end{array}$ & $\begin{array}{c}\text { EUV }+193 \mathrm{~nm} \\
\mathrm{CD}=27.2 \mathrm{~nm} \\
\mathrm{LER}=6.64 \mathrm{~nm}\end{array}$ & $\begin{array}{c}\text { EUV }+248 \mathrm{~nm} \\
\mathrm{CD}=28.0 \mathrm{~nm} \\
\mathrm{LER}=5.42 \mathrm{~nm}\end{array}$ \\
\hline CD $=29.0 \mathrm{~nm}$ \\
$\mathrm{LER}=5.3 \mathrm{~nm}$
\end{tabular}

Figure 10: Average LER computed for BBR08A for the 30nm half-pitch. Significant increase in LER is observed with OOB exposure. 


\subsection{OOB effects on BBR08B}

The same measurement methodology was repeated for BBR08B. 50-nm half-pitch line and space process performance is compared for the UV exposure environment vs standard conditions (Fig. 11). Differences observed are within the quantified error bars for all process metrics.

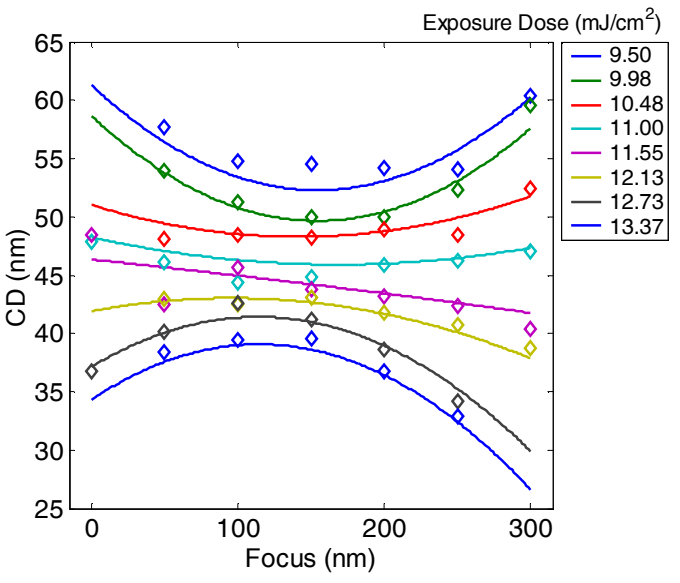

EUV only process window

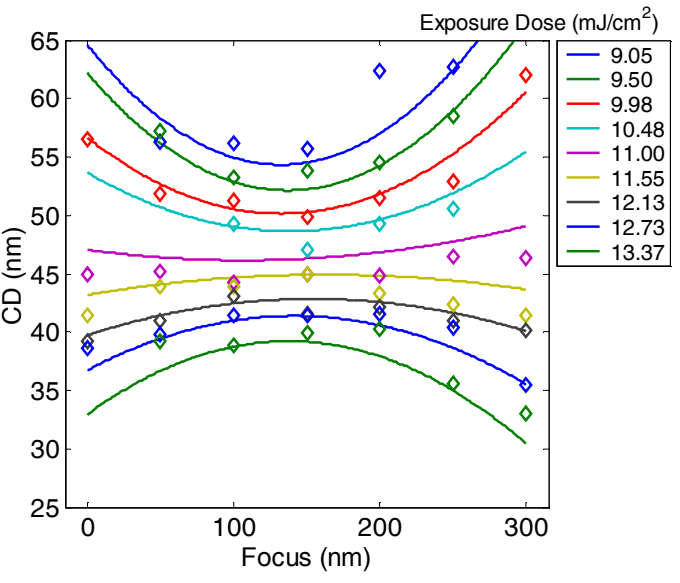

$1.00 \mathrm{~mJ} / \mathrm{cm}^{2}$ uniform Broad-band+EUV

Figure 11. Through focus and dose process distribution for standard EUV patterning (left) vs. OOB exposure environment exposed resist fim (right). Results show only differences within the quantified error bar for all process metrics.
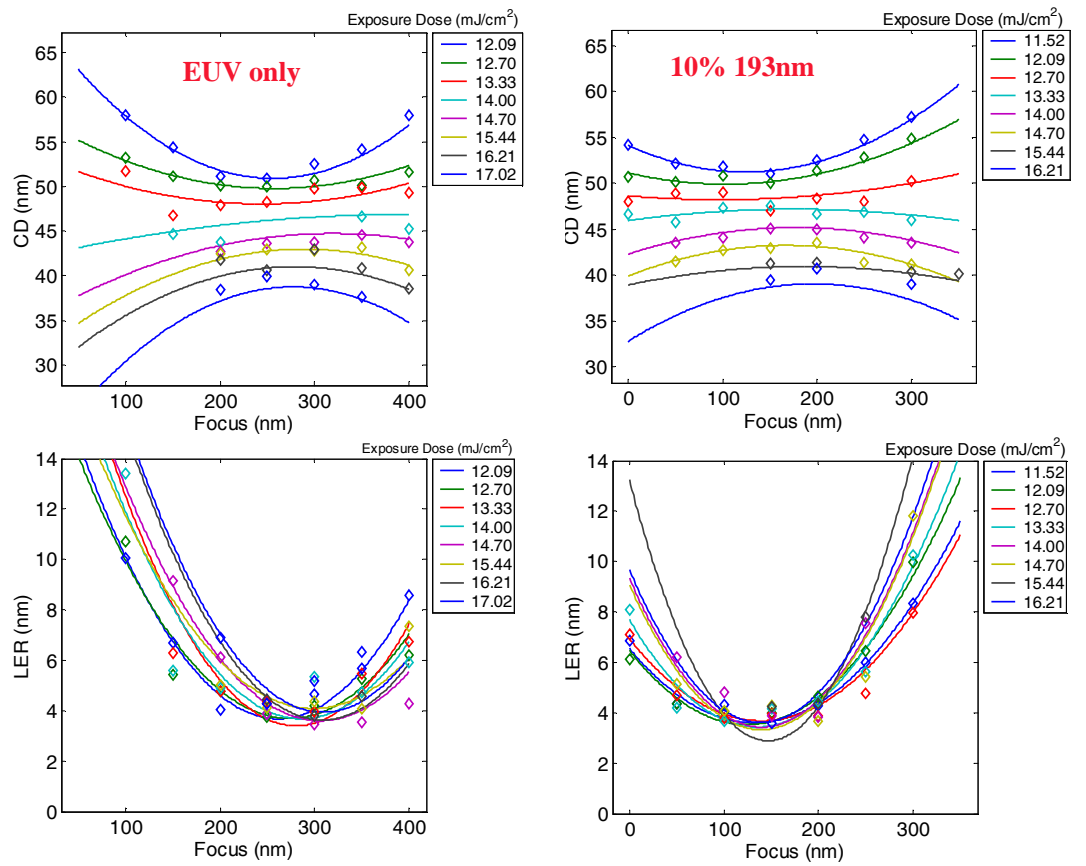

EUV only, $\mathrm{E}_{50}=12.70 \mathrm{~mJ}, \mathrm{EL}=28 \%$, $\mathrm{DOF}=293 \mathrm{~nm}, \mathrm{LER}=4.6 \mathrm{~nm}$

$193 \mathrm{~nm}, 0.66 \mathrm{~mJ} / \mathrm{cm}^{2}, \mathrm{E}_{50}=12.09 \mathrm{~mJ}$ $\mathrm{EL}=26 \%, \mathrm{DOF}=350 \mathrm{~nm}, \mathrm{LER}=4.00 \mathrm{~nm}$
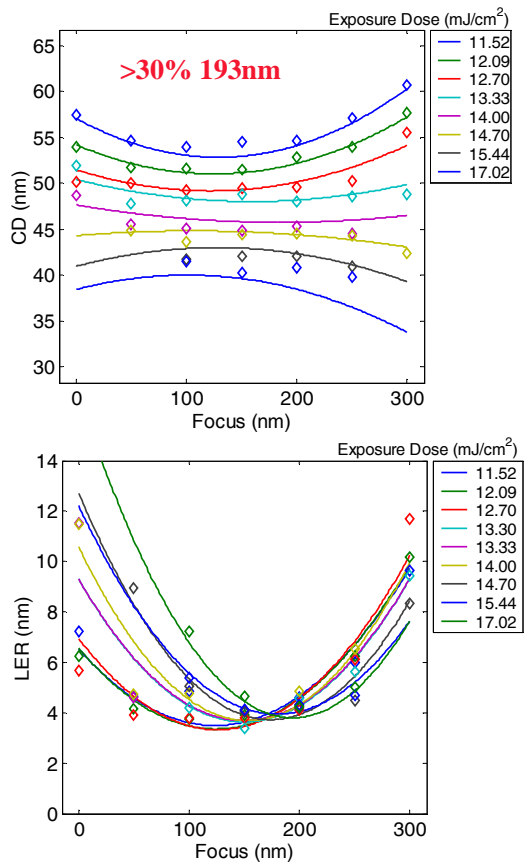

$193 \mathrm{~nm}, 3.03 \mathrm{~mJ} / \mathrm{cm}^{2}, \mathrm{E}_{50}=12.7 \mathrm{~mJ}$, $\mathrm{EL}=28 \%, \mathrm{DOF}=300 \mathrm{~nm}, \mathrm{LER}=3.93 \mathrm{~nm}$

Figure 12. Through focus and dose process distribution for standard EUV patterning (left) vs. OOB exposure environment exposed resist film (right). Results show only differences within the quantified error bar for all process metrics.

Figure 12 shows the corresponding LER through focus An average LER of $4.0 \mathrm{~nm}$ was found for $10 \%$ OOB exposure, while the EUV only patterning gave an average LER of $4.6 \mathrm{~nm}$. The reason behind the improved process is not 
understood at this time. Further examination of the resolution performance (Fig. 13) is needed as well. Rigorous examination of the induced chemical changes in the film as a result of UV exposure needs to be better understood.

Resist B with BARC: 28nm HP
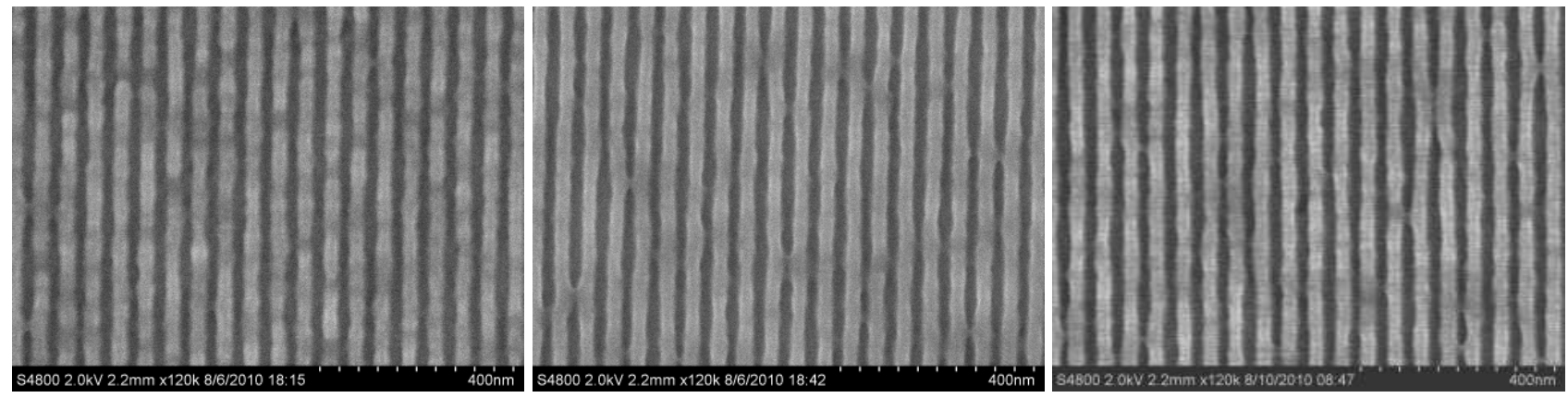

$$
\begin{gathered}
\text { Reference } \\
C D=29.6 \mathrm{~nm} \\
\text { LER }=6.29 \mathrm{~nm}
\end{gathered}
$$

$$
\begin{gathered}
\mathrm{EUV}+193 \mathrm{~nm} \\
\mathrm{CD}=30.5 \mathrm{~nm} \\
\mathrm{LER}=4.82 \mathrm{~nm}
\end{gathered}
$$

Figure 13. $3 \sigma$ LER measurements corresponding to the data shown in figure 10 is given. (Left) EUV only imaging, (Right) EUV patterning over a broadband illumination dose of $1 \mathrm{~mJ} / \mathrm{cm}^{2}$, average LER of $5.2 \mathrm{~nm}$ was found for the best conditions for the $28 \mathrm{~nm}$ half-pitch close to the resolution limit.

\section{SUMMARY AND DISCUSSION}

In this paper, an overview of the previous works for the first order estimation of the effective flare from the resist sensitive OOB wavelengths is given. We show that the OOB-related flare is resist specific due to wavelength sensitivity. The true impact of OOB on mask is shown to vary with pattern size, shape, density, mask tone, and absorber characteristics.

Results from the first set of controlled OOB experiments are also presented. The results show an impact on LER and on process performance. One of the resists evaluated is shown to have an improved process performance. Possible chemical changes induced by VUV light may involve the removal of lactone and ester groups in the resist and changes in the glass transition temperature induced by VUV heating. Efforts are underway for a more rigorous and complete experimental evaluation of multiple resists.

\section{ACKNOWLEDGMENTS}

The authors would like to acknowledge the support and dedication of the CXRO engineering team and staff. Special thanks to the MET team members. Mirror reflectance and optical constants were provided by Dr. Eric Gullikson. We would like to acknowledge the Energetiq team, especially Debbie Gustafson for providing support and source spectral characterizations for this work. Thomas I. Wallow of Global Foundries was instrumental providing guidance and support for these studies. Helpful discussions with Todd Younkin, Robert Sulc, James Blackwell from Intel and Jan Lorusso from IMEC are acknowledged. The SEMATECH Berkeley MET is supported by SEMATECH. This work was supported by the Director, Office of Science, Office of Basic Energy Sciences, of the U.S. Department of Energy under Contract No. DE-AC02-05CH11231. 


\section{REFERENCES}

[1] Bakshi, V., ed., "EUV Sources for Lithography," SPIE Press, Washington (2005)

[2] Gullikson, E., "Multilayer reflectivity," (2007).

[3] Palik, E. D. and Ghosh, G., Handbook of Optical Constants of Solids, Academic Press, San Diego, CA (1997). http://henke.lbl.gov/optical_constants.

[4] Roberts, J. M. Bristol, R. L., Younkin, T. R., Fedynyshyn, T. H., Astolfi, D. K., and Cabral, A., "Sensitivity of EUV resists to out-of-band radiation,” Proc. SPIE 7273, 72731W (2009).

[5] George, S. A., Naulleau, P. P., Rekawa, S., Gullikson, E., and Kemp, C. D., "Estimating the out-of-band radiation flare levels for extreme ultraviolet lithography," J. Micro/Nanolith. MEMS MOEMS 8, 041502 (2009)

[6] Naulleau, P. P., Anderson, C. N., Chiu, J., Dean, K., Dehnam, P. E., George, S. A., Goldberg, K. A., Hoef, B. H., Jones, G., Koh, C., La Fontaine, B., Ma, A., Montgomery, W., Niakoula, D., Park, J.-O., Wallow, T. I., Wurm, S. "Latest results from the SEMATECH Berkeley extreme ultraviolet mircofield exposure tool," JVST B, 27, p. 66, (2009)

[7] Naulleau, P. P., Gullikson, E. M., Aquila, A., George, S., and Niakoula, D., Absolute sensitivity calibration of extreme ultraviolet photoresists," Opt. Express 16(15), 11519-11524 (2008)

[8] Miyake, A., Kanazawa, H., Banine, V., and Suzuki, K., Joint requirements," Presentation at EUV Work-shop, Proceedings available at www.sematech.org (October 19. 2006).

[9] Mizuno, H., McIntyre, G., Koay, C-S., Burkhardt, M., LaFontaine, B., and Wood, O., "Flare evaluation of ASML alpha demo tool," Proc. SPIE 7271, 72710U (2009)

[10] Panoramic Technology, "EM-Suite/Hyperlith," http://www.panoramictech.com (2009).

[11] George, S. A., Naulleau, P. P., "Assessing out-of-band flare effects at the wafer level for EUV lithography", Proc. SPIE 7636, 763626 (2010).

[12] LDLS ${ }^{\text {TM }}$ Laser-Driven Light Sources, http://www.energetiq.com/html/ldls.html

[13] Acton Optics \& Coatings, "Optical filters," (2009). http://www.princetoninstruments. com/products/optics/filters/default.aspx\#1.

[14] SXUV Series Photodiodes, http://www.ird-inc.com/sxuv.html

[15] Hitachi High-Technologies, http://www.hht-eu.com/cms/3171.html

[16]EUV Technology SuMMIT Software Division, http://www.lithometrix.com

\section{DISCLAIMER}

This document was prepared as an account of work sponsored by the United States Government. While this document is believed to contain correct information, neither the United States Government nor any agency thereof, nor The Regents of the University of California, nor any of their employees, makes any warranty, express or implied, or assumes any legal responsibility for the accuracy, completeness, or usefulness of any information, apparatus, product, or process disclosed, or represents that its use would not infringe privately owned rights. Reference herein to any specific commercial product, process, or service by its trade name, trademark, manufacturer, or otherwise, does not necessarily constitute or imply its endorsement, recommendation, or favoring by the United States Government or any agency thereof, or The Regents of the University of California. The views and opinions of authors expressed herein do not necessarily state or reflect those of the United States Government or any agency thereof or The Regents of the University of California. 